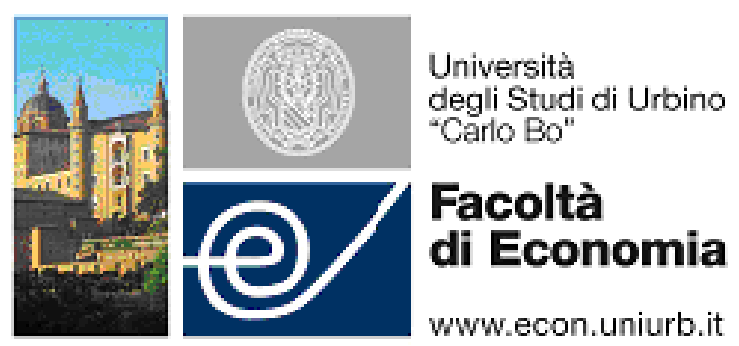

WP-EMS

Working Papers Series in Economics, Mathematics and Statistics

\title{
“A PIECEWISE LINEAR MODEL OF CREDIT TRAPS AND CREDIT CYCLES: A COMPLETE CHARACTERIZATION"
}

- Kiminori Matsuyama (Northwestern University, USA)

- Iryna Sushko (Institute of Mathematics, National Academy of Science of Ukraine)

- Laura Gardini (Department of Economics, Society and Politics, University of Urbino) 


\title{
A Piecewise Linear Model of Credit Traps and Credit Cycles: A Complete Characterization
}

\author{
Kiminori Matsuyama ${ }^{a}$, Iryna Sushko $^{b}$, Laura Gardini $^{c}$ \\ ${ }^{a}$ Northwestern University, USA, \\ ${ }^{b}$ Institute of Mathematics, National Academy of Science of Ukraine, \\ ${ }^{c}$ University of Urbino, Italy
}

\begin{abstract}
We reconsider a regime-switching model of credit frictions which has been proposed in a general framework by Matsuyama for the case of CobbDouglas production functions. This results in a piecewise linear map with two discontinuity points and all three branches having the same slope. We offer a complete characterization of the bifurcation structure in the parameter space, as well as of the attracting sets and related basins of attraction in the phase space. We also discuss parameter regions associated with overshooting, leapfrogging, poverty traps, reversal of fortune, and growth miracle, as well as cycles with any kind of switching between the expansionary and contractionary phases.
\end{abstract}

Keywords: Macroeconomic model of credit frictions; Poverty traps; Growth miracle; One-dimentional piecewise linear map; Border collision bifurcation

\section{Introduction}

How does the frictions in the credit market affect the macro dynamics? To answer this question in a simple manner, Matsuyama (2007) proposed a regimeswitching model of credit frictions. The key feature of the model is that entrepreneurs have access to different types of investment projects, and yet, only one type of investment projects is financed at each state of the economy, and the credit flow abruptly switches from one type to another as the state of the economy changes. Due to this regime-switching nature, this model generates a rich array of dynamic behavior. However, a complete characterization of the dynamic behavior was lacking in Matsuyama (2007). In the present paper, we study this model for the case of Cobb-Douglas production functions, which makes the dynamic system characterized by a piecewise linear map with two 
discontinuity points. This allows us to offer a complete characterization of its dynamic behaviors for a full set of the parameter space.

The dynamics of piecewise linear maps with one discontinuity point are well known in the case of slopes all smaller than one in modulus (see Leonov 1959; Leonov 1962; Keener 1980; Avrutin et al. 2010; Gardini et al. 2010, and applications to economics in Gardini et al. 2011; Tramontana et al. 2010; Tramontana et al. 2011), and this is also relevant to our system. However, our system has two discontinuity points, which can be seen, for example, in Tramontana et al. 2011. The restrictions related to the economic significance of the model, lead to equality of two of the three branches of the map, and this particularity allows us a complete characterization of the dynamics of the model.

The structure of the paper is as follows. In Sec.2 we recall the regime switching model of credit frictions proposed by Matsuyama (2007), which generates a piecewise smooth system in a general setup. We go further and provide a complete characterization under the additional assumptions that the agents have access to two types of investment projects and that the final goods production technology is Cobb-Douglas, $y_{t}=f\left(k_{t}\right)=A\left(k_{t}\right)^{\alpha}$, with $0<\alpha<1$. In this case the system can be rewritten in a piecewise linear form with two discontinuity points, which can be analytically investigated in detail. This is done in Sec.3. In Sec.3.1 we describe the case where there is a unique, globally attracting steady state, and yet overshooting and leapfrogging can occur, depending on the parameters, while in Sec.3.2, due to the coexistence of two stable steady states, we show how poverty traps and reversal of fortune can occur.

Section 3.3 considers the case in which the system has no steady states, and in the absorbing interval the system reduces to a piecewise linear map with two branches whose slopes are both positive and smaller than 1 . In this case, the economy system has a unique credit cycle, which is globally attracting. Due to its simplicity, the nature of such globally attracting credit cycle can be completely characterized with analytical expressions of the border collision bifurcation curves. It is well-known that in piecewise linear maps of this kind the only possible bifurcations are related to the discontinuity point. The bifurcations leading to the appearance and disappearance of the cycle are characterized by the collision (or merging) of a periodic point of the cycle with the discontinuity point from the two opposite sides (left/right). So stable cycles of any period can emerge, although Cantor set attractors (structurally unstable) may also exist, but in a set of parameter values of zero Lebesgue measure. The periodicity regions related to the existence of cycles in the parameter space follow the period adding bifurcation structure, which means that rotation numbers of the corresponding cycles follow the Farey summation rule. That is, between two periodicity regions of cycles with rotation numbers $\frac{p_{1}}{k_{1}}$ and $\frac{p_{2}}{k_{2}}$ there exists the periodicity region of cycles with the rotation number given by $\frac{p_{1}}{k_{1}} \oplus \frac{p_{2}}{k_{2}}=\frac{p_{1}+p_{2}}{k_{1}+k_{2}}$, where $\oplus$ denotes the operation known as Farey summation rule. The rotation number associated with the existing cycle and the related symbolic sequence, show how the economy alternates between the expansionary and contractionary 
phases. We describe how asymmetry of cycles (the fraction of time the economy is in the expansionary phase) varies with the credit frictions parameters. In Sec.3.4 we consider the cases in which the steady state may be the unique attractor or it may coexist with a stable cycle of any period. The latter case is further divided into two cases, the cycle as a poverty trap and growth miracle, where the economy may fluctuate for a long time at a lower level before successfully escaping from the poverty. Section 4 concludes. As already remarked, what simplifies the analysis is that the functions defining the map are linear contractions. However, qualitatively similar results can be analytically obtained, with implicit equations, and numerically justified, for the system described by a discontinuous piecewise smooth function.

\section{A regime switching model of credit frictions: A quick review of Matsuyama (2007)}

The economy produces a single final good, using the CRS technology, $Y_{t}=$ $F\left(K_{t}, L_{t}\right)$, where $K_{t}$ is physical capital, and $L_{t}$ is labor. The final good produced in period $t$ may be consumed or allocated to investment projects. Let $y_{t} \equiv$ $Y_{t} / L_{t}=F\left(k_{t}, 1\right) \equiv f\left(k_{t}\right)$, where $k_{t}=K_{t} / L_{t}$, and $f(k)$ satisfies $f^{\prime}(k)>0>$ $f^{\prime \prime}(k)$. The markets are competitive, and the factor rewards for physical capital and for labor are $\rho_{t}=f^{\prime}\left(k_{t}\right)$ and $w_{t}=f\left(k_{t}\right)-k_{t} f^{\prime}\left(k_{t}\right) \equiv W\left(k_{t}\right)>0$, which are both paid in the final good. For simplicity, physical capital is assumed to depreciate fully in one period.

In each period, a new generation of potential entrepreneurs, a unit measure of homogeneous agents, arrives with one unit of the endowment, called labor. They stay active for two periods, as in the Diamond overlapping generations model (see Diamond 1965). In the first period, they sell the labor endowment and earn $w_{t}=W\left(k_{t}\right)$. They consume only in the second period. Thus, they save all of the earning, $w_{t}$, and allocate it to maximize their second period consumption. They may become lenders or entrepreneurs. If they become lenders, they can earn the gross return equal to $r_{t+1}$ per unit in the competitive credit market and consume $r_{t+1} w_{t}$ in the second period. Alternatively, they may become entrepreneurs by using their earning, $w_{t}$, to partially finance an investment project. They can choose from $J$ different types of projects. All projects come in discrete, indivisible units and each entrepreneur can run only one project. A type- $j(j=1,2, \ldots, J)$ project transforms $m_{j}$ units of the final good in period $t$ into $m_{j} R_{j}$ units of physical capital in period $t+1$. Because of the fixed investment size, $m_{j}$, an entrepreneur needs to borrow by $m_{j}-w_{t}$ at the rate equal to $r_{t+1}$.

Let $X_{j t}$ denote the measure of type- $j$ projects initiated in period $t$. Then, the aggregate investment, the amount of the final good allocated to all the projects, is $I_{t}=\sum_{j=1}^{J} m_{j} X_{j t}$. Since the aggregate saving is $S_{t}=W\left(k_{t}\right)$, the credit market 
equilibrium requires that

$$
W\left(k_{t}\right)=\sum_{j=1}^{J} m_{j} X_{j t}
$$

The capital stock adjusts according to

$$
k_{t+1}=\sum_{j=1}^{J} m_{j} R_{j} X_{j t}
$$

Let us now turn to the investment decisions. To invest in a project, the entrepreneurs must be both willing and able to borrow. By becoming the lenders, the agents can consume $r_{t+1} w_{t}$. By running type- $j$ projects, they can consume $m_{j} R_{j} \rho_{t+1}-r_{t+1}\left(m_{j}-w_{t}\right)$. Thus, the agents are willing to borrow and to run a type- $j$ project if and only if

$$
m_{j} R_{j} \rho_{t+1}-r_{t+1}\left(m_{j}-w_{t}\right) \geq r_{t+1} w_{t}
$$

which can be simplified to

$$
(\mathrm{PC}-j): \quad R_{j} f^{\prime}\left(k_{t+1}\right) \geq r_{t+1}
$$

where PC stands for the profitability constraint.

Even when $(\mathrm{PC}-j)$ holds, the agents may not be able to invest in type- $j$ projects, due to the borrowing constraint. The borrowing limit exists because borrowers can pledge only up to a fraction of the project revenue for the repayment, $\lambda_{j} m_{j} R_{j} \rho_{t+1}$, where $0<\lambda_{j}<1$. Knowing this, the lender would lend only up to $\lambda_{j} m_{j} R_{j} \rho_{t+1} / r_{t+1}$. Thus, the agent can borrow to run a type- $j$ project if and only if

$$
(\mathrm{BC}-j): \quad \lambda_{j} m_{j} R_{j} f^{\prime}\left(k_{t+1}\right) \geq r_{t+1}\left[m_{j}-W\left(k_{t}\right)\right]
$$

where BC stands for the borrowing constraint.

Suppose that $R_{j} f^{\prime}\left(k_{t+1}\right)>r_{t+1} \max \left\{1, \frac{m_{j}-W\left(k_{t}\right)}{\lambda_{j} m_{j}}\right\}$, so that both (PC- $j$ ) and $(\mathrm{BC}-j)$ are satisfied with strict inequalities. Then, any agent would be able to borrow and run a type- $j$ project and would be better off by doing so than by lending. This means that no agent would become a lender. Hence, in equilibrium, $R_{j} f^{\prime}\left(k_{t+1}\right) \leq r_{t+1} \max \left\{1, \frac{m_{j}-W\left(k_{t}\right)}{\lambda_{j} m_{j}}\right\}$ must hold. If this inequality holds strictly for some $j$, then at least one of $(\mathrm{PC}-j)$ and $(\mathrm{BC}-j)$ is violated, so that $X_{j t}=0$. Since (1) requires that $X_{j t}>0$ for some $j$, we have

$$
\frac{r_{t+1}}{f^{\prime}\left(k_{t+1}\right)}=\max _{j}\left\{\frac{\lambda_{j} R_{j}}{\max \left\{\lambda_{j}, 1-\frac{W\left(k_{t}\right)}{m_{j}}\right\}}\right\} \geq \frac{\lambda_{j} R_{j}}{\max \left\{\lambda_{j}, 1-\frac{W\left(k_{t}\right)}{m_{j}}\right\}}
$$

The RHS of (4), multiplied by $f^{\prime}\left(k_{t+1}\right)$, is the rate of return that the lenders could expect from lending to the agents running project- $j$. Because the credit 
flows only to the project that generates the highest rate of return to the lender, $X_{j t}>0(j=1,2, \ldots, J)$ only if the inequality in (4) holds with the equality.

Notice that, for each $k_{t}$, we can rank the projects by the RHS of (4). Thus, generically, there exists only one type of project, $J\left(k_{t}\right)$, for which the inequality in (4) holds with the equality, and all the credit flows to this project. That is, $X_{j t}=1$ if $j=J\left(k_{t}\right)$, and $X_{j t}=0$, otherwise. This means that eqs. (1)-(4) are simplified to:

$$
k_{t+1}=R_{J\left(k_{t}\right)} W\left(k_{t}\right)
$$

For any $k_{0}>0$, we can determine the equilibrium trajectory of this economy by iterating (5).

Below, we focus on the case considered in Matsuyama (2007) (Sec.4), where the following inequalities hold:

$$
R_{2}>R_{1}>\lambda_{2} R_{2}>\lambda_{1} R_{1}
$$

and

$$
\frac{m_{2}}{m_{1}}>\frac{1-\lambda_{1}}{1-\lambda_{2} \frac{R_{2}}{R_{1}}}>1
$$

Fig.1 illustrates the implications of these parameter restrictions with the graphs of

$$
\frac{\lambda_{j} R_{j}}{\max \left\{\lambda_{j}, 1-\frac{W\left(k_{t}\right)}{m_{j}}\right\}} \text { for } j=1,2
$$

- Because Project 1 is less productive than Project $2\left(R_{2}>R_{1}\right)$, Project 2 dominates Project 1 when $W\left(k_{t}\right)>W\left(k_{c c}\right)$ for $k_{t}>k_{c c}$.

- Because Project 1 is less pledgeable than Project $2\left(\lambda_{2} R_{2}>\lambda_{1} R_{1}\right)$, Project 2 dominates Project 1 , when $W\left(k_{t}\right)<W\left(k_{c}\right)$ or $k_{t}<k_{c}$.

- Because the setup cost of Project 1 is sufficiently smaller than that of Project $2\left(m_{2} / m_{1}>\left(1-\lambda_{1}\right) /\left(1-\lambda_{2} R_{2} / R_{1}\right)>1\right)$, and because Project 1 is sufficiently productive $\left(\lambda_{2} R_{2}<R_{1}\right)$, Project 1 dominates Project 2 for $W\left(k_{t}\right) \in$ $\left(W\left(k_{c}\right), W\left(k_{c c}\right)\right)$, or $k_{c}<k_{t}<k_{c c}$.

With all the credit flowing into Project 1 for $k_{c}<k_{t}<k_{c c}$, and all the credit flowing into Project 2 for $k_{t}<k_{c}$ or $k_{t}>k_{c c}$, (5) becomes:

$$
k_{t+1}= \begin{cases}R_{2} W\left(k_{t}\right) & \text { if } k_{t}<k_{c} \text { or } k_{t}>k_{c c} \\ R_{1} W\left(k_{t}\right) & \text { if } k_{c}<k_{t}<k_{c c}\end{cases}
$$

Since $R_{2}>R_{1}$, the map defined in (6) jumps down as $k_{t}$ passes $k_{c}$ and jumps up as $k_{t}$ passes $k_{c c}$. The intuition should be clear. When the net worth is very low, the entrepreneurs must rely almost entirely on external finance, so that the saving flows into type- 2 projects that generate more pledgeable return per unit of investment. As the net worth rises, the entrepreneurs can offer more attractive rate of return with type- 1 projects than with type- 2 projects, because they need to borrow little for type- 1 projects. Hence, a rise in the net worth leads to a shift of the credit toward less productive projects. If the net worth 


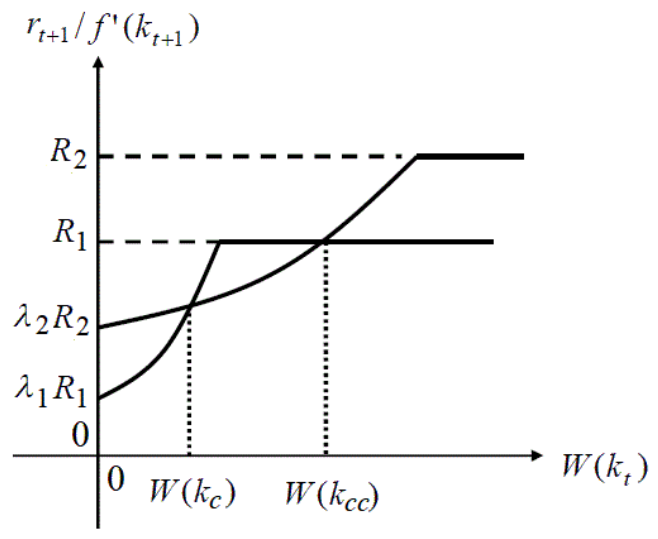

Figure 1: Qualitative graphs, resulting from the parameter restrictions.

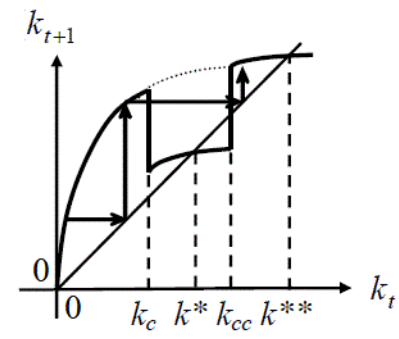

(a)

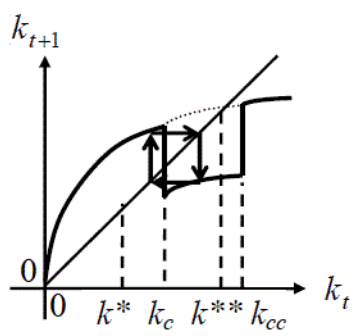

(b)

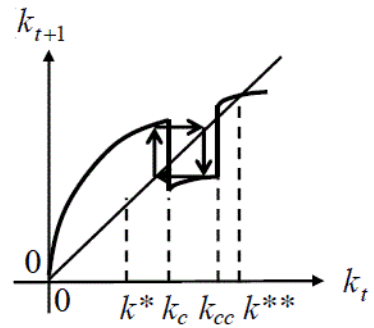

(c)

Figure 2: (a) Credit trap, or leapfrogging, or reversal of fortune; (b) credit cycles; (c) cycles as a trap or growth miracle.

rises even further, then the borrowing need becomes small enough for type-2 projects that the credit shifts back to more productive type- 2 projects.

Figures 2a-2c illustrate some possibilities generated by $(6)$ when $W\left(k_{t}\right)$ is concave. In these figures, $k^{*}>0$, defined uniquely by $k^{*} \equiv R_{1} W\left(k^{*}\right)$, would be the steady state of the economy, when the credit would always go to the less productive Project-1 and $k^{* *}\left(>k^{*}\right)$, defined uniquely by $k^{* *} \equiv R_{2} W\left(k^{* *}\right)$, would be the steady state of the economy, when the credit would always go to the more productive Project- 2 .

Figure 2a shows the case where $k_{c}<k^{*}<k_{c c}<k^{* *}$. In this case, two locally stable steady states, $k^{*}$ and $k^{* *}$, coexist. When the economy starts from $k_{0}>k_{c c}$, it always converges monotonically to the higher steady state, $k^{* *}$. The lower steady state $k^{*}$ is a credit trap, to which the economy converges monotonically, when it starts from $k_{0} \in\left(k_{c}, k_{c c}\right)$. What happens when the economy starts from $k_{0}<k_{c}$ ? There are three cases to be distinguished. If $R_{2} W\left(k_{c}\right)<k^{*}$, the economy always converges monotonically to $k^{*}$. If $k^{*}<$ 
$R_{2} W\left(k_{c}\right)<k_{c c}$, the economy converges to $k^{*}$, but the convergence may not be monotonic. Instead, it may overshoot $k^{*}$, and then converges towards it from above. If $R_{2} W\left(k_{c}\right)>k_{c c}$, however, it may manage to bypass the region $\left(k_{c}, k_{c c}\right)$ and converge to the higher steady state, $k^{* *}$, as illustrated by the arrows in the figure. In particular, a country that starts below the other countries could end up overtaking them, creating the possibility of leapfrogging and reversal of fortune.

Figure $2 \mathrm{~b}$ shows the case where $k^{*}<k_{c}<k^{* *}<k_{c c}$. In this case, there is no steady state, to which the equilibrium trajectory would converge to. Instead, the trajectory always converges to a credit cycle, which fluctuates around $k_{c}$, within the interval $\left(R_{1} W\left(k_{c}\right), R_{2} W\left(k_{c}\right)\right)$.

Figure 2c shows the case where $k^{*}<k_{c}<k_{c c}<k^{* *}$. In this case, a credit cycle coexist with the high steady state, $k^{* *}$. Then, this case may be viewed as a hybrid of the cases depicted in Fig.2a and Fig.2b. Starting from $k_{0}<k_{c c}$, the economy will be trapped into the credit cycle below $k_{c c}$ if $R_{2} W\left(k_{c}\right)<k_{c c}$. If $R_{2} W\left(k_{c}\right)>k_{c c}$, on the other hand, it may achieve a growth miracle, by managing to escape and converge to the high steady state, $k^{* *}$, after fluctuating below $k_{c c}$ possibly for many periods.

Although Matsuyama (2007) discussed how such a rich set of dynamic behaviors can occur in this model, he came short of providing a complete analysis. In particular, he did not offer any characterization of the nature of credit cycles, such as the period length or asymmetry, when they exist.

\section{Analysis of the dynamics}

As remarked in the Introduction, in this paper we go further and provide a complete characterization under the additional assumption of a Cobb-Douglas technology, $y_{t}=f\left(k_{t}\right)=A\left(k_{t}\right)^{\alpha}$, with $0<\alpha<1$. In this case, (6) can be rewritten in the following piecewise linear form:

$$
x_{t+1}=\left\{\begin{array}{ll}
\theta_{2}+\alpha x_{t} & \text { if } x_{t}<d_{c} \text { or } x_{t}>d_{c c} \\
\theta_{1}+\alpha x_{t} & \text { if } d_{c}<x_{t}<d_{c c}
\end{array}\right. \text {. }
$$

by defining $x_{t} \equiv \log _{b}\left(k_{t}\right) ; \theta_{1} \equiv \log _{b} R_{1}(1-\alpha) A<\theta_{2} \equiv \log _{b} R_{2}(1-\alpha) A$ and $d_{c} \equiv \log _{b}\left(k_{c}\right)<d_{c c} \equiv \log _{b}\left(k_{c c}\right)$. Because there are three goods (the final good, capital, and labor) in this model, there are two degrees of freedom in choosing the units of measurement. We do so by setting

$$
A=\frac{1}{R_{1}(1-\alpha)} ; \quad b=\left(\frac{R_{2}}{R_{1}}\right)^{1 /(1-\alpha)}>1
$$

so that $\theta_{1}=0$ and $\theta_{2}=1-\alpha$. Then,

$$
x_{t+1}= \begin{cases}(1-\alpha)+\alpha x_{t} & \text { if } x_{t}<d_{c} \text { or } x_{t}>d_{c c} \\ \alpha x_{t} & \text { if } d_{c}<x_{t}<d_{c c}\end{cases}
$$


where the two discontinuity (or switching) points, $d_{c}<d_{c c}$, are given by:

$$
\alpha d_{c} \equiv \log _{b}\left(\frac{R_{1} m_{2}\left(\frac{\lambda_{2}}{\lambda_{1}} \frac{R_{2}}{R_{1}}-1\right)}{\frac{\lambda_{2}}{\lambda_{1}} \frac{R_{2}}{R_{1}} \frac{m_{2}}{m_{1}}-1}\right) ; \alpha d_{c c} \equiv \log _{b}\left(R_{1} m_{2}\left(1-\lambda_{2} \frac{R_{2}}{R_{1}}\right)\right)
$$

Notice that, if all the credit always flowed to the less productive type-1 projects, $x_{t+1}=\alpha x_{t}$, hence $x_{t}$ converges monotonically to $x^{*}=0$. If all the credit always flowed to the more productive type- 2 projects, $x_{t+1}=(1-\alpha)+\alpha x_{t}$, hence $x_{t}$ converges monotonically to $x^{* *}=1$. However, the credit frictions can cause the credit flow to switch between the two projects, which affects dynamics. The parameters that capture credit frictions, $\left(\lambda_{1}, m_{1}, \lambda_{2}, m_{2}\right)$ affect dynamics through a change in the switching points, $\left(d_{c}, d_{c c}\right)$.

We now turn to a detailed analysis of (8) to see how the dynamics depend on $\left(d_{c}, d_{c c}\right)$.

Let the map given in (8) be denoted as $g$, and for our convenience let us rewrite it in the following form:

$$
g: x_{t+1}=g\left(x_{t}\right)= \begin{cases}g_{L}\left(x_{t}\right)=(1-\alpha)+\alpha x_{t} & \text { if } x_{t}<d_{c} \\ g_{R}\left(x_{t}\right)=\alpha x_{t} & \text { if } d_{c}<x_{t}<d_{c c} \\ g_{U}\left(x_{t}\right)=(1-\alpha)+\alpha x_{t} & \text { if } x_{t}>d_{c c}\end{cases}
$$

As we shall see, the upper branch of the map, denoted $g_{U}(x)$, is involved into the asymptotic dynamics only when its fixed point $x^{* *}=1$ exists (and it is attracting), otherwise, the absorbing interval involves only the branches $g_{L}(x)$ and $g_{R}(x)$, and in such a case attracting cycles of different periods may exist.

In this section we describe the dynamic behaviors of map $g$ given in (9). To be used below, the two linear functions $g_{L}$ and $g_{R}$ have a simple expression for the inverses:

$$
g_{L}^{-n}(z)=\frac{z}{\alpha^{n}}+1-\frac{1}{\alpha^{n}}, \quad g_{R}^{-n}(z)=\frac{z}{\alpha^{n}}, \forall n \geq 1
$$

For each fixed value of the parameter $\alpha, 0<\alpha<1$, of the Cobb-Douglas production function we can describe the dynamics as a function of the two parameters $d_{c}$ and $d_{c c}$, that is, we can investigate the bifurcation structure of the parameter plane $\left(d_{c}, d_{c c}\right)$. The overview of the results is shown in Fig.3 for $\alpha=0.3<0.5$ and $\alpha=0.7>0.5$, where different colors are used to denote the existence regions of different attracting cycles.

In the $\left(d_{c}, d_{c c}\right)$-parameter plane only the range $d_{c}<d_{c c}$ is of interest (that is, above the main diagonal of the parameter plane denoted $(d)$ in Fig.3), and in that half plane we can distinguish several regions associated with the qualitatively similar dynamics described in the next subsections. In fact, we have three regions denoted S-I, S-II and S-III, which are related to rather simple situations: any trajectory converges to a unique global attractor, with different kinds of transients. The boundaries of these regions are related to the appearance/disappearance of a fixed point via a border collision bifurcation (BCB for short henceforth). In general, not only for the fixed points but for any cycle, 

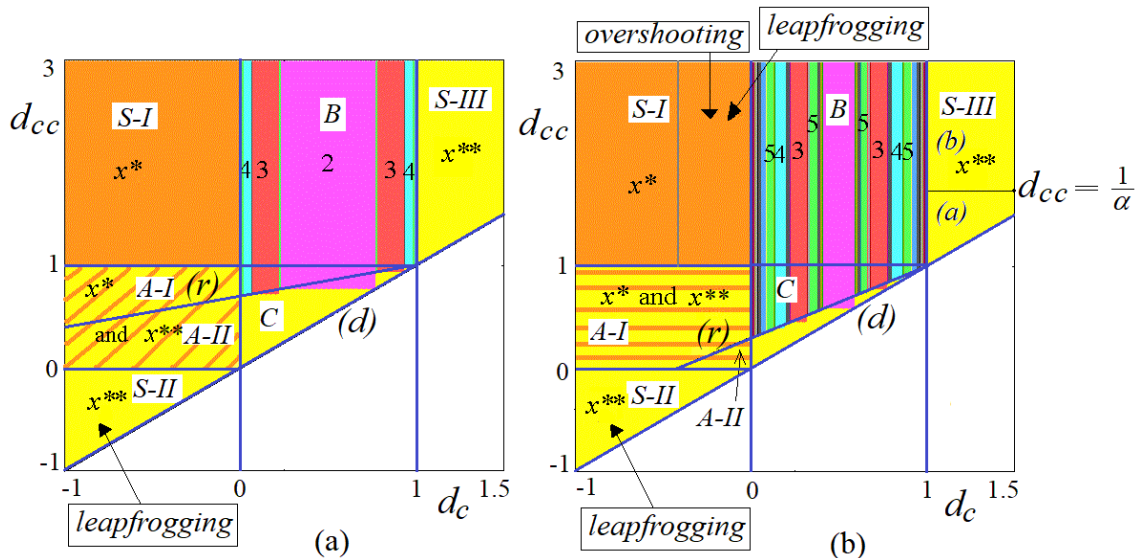

Figure 3: 2D bifurcation diagram in the $\left(d_{c}, d_{c c}\right)$-parameter plane of map $g$ at $\alpha=0.3$ (a) and $\alpha=0.7$ (b).

in piecewise linear maps in which the slopes are smaller than 1 in modulus, all the existing cycles can only be attracting, and the appearance/disappearance of a cycle can only occur via collision with a border point, that is, whenever a periodic point of a cycle collides with one of the two discontinuity points of the map, as stated in the following

Property (Stability of cycles) The dynamics of map g given in (9), are always bounded in an absorbing interval. All the $k$-cycles, $k \geq 1$, which may exist are locally asymptotically stable. The appearance/disappearance of an attracting cycle can occur only via border collision bifurcation, when one periodic point collides with one discontinuity point.

In particular, for the case $k=1$, it is immediate to verify that the fixed point $x^{*}=0$ exists iff $d_{c}<0$ and $d_{c c}>0$. Differently, the fixed point $x^{* *}=1$ exists iff $g_{L}\left(d_{c}\right)<d_{c}$ or $g_{U}\left(d_{c c}\right)>d_{c c}$ leading to the conditions $d_{c}>1$ or $d_{c c}<1$. We can so state the following

Proposition 1 (BCB of fixed points) Let $d_{c}<d_{c c}$. In the $\left(d_{c}, d_{c c}\right)$-parameter plane the straight lines defined by $d_{c}=0$ and $d_{c c}=0$ are $B C B$ curves related to the appearance/disappearance of the fixed point $x^{*}=0$, so that the fixed point $x^{*}$ exists for $d_{c}<0$ and $d_{c c}>0$; the straight lines defined by $d_{c}=1$ and $d_{c c}=1$ are $B C B$ curves related to the appearance/disappearance of the fixed point $x^{* *}=1$ so that the fixed point $x^{* *}$ exists for $d_{c}>1$ or $d_{c c}<1$.

The straight lines mentioned in Proposition 1 bound the three regions with simple dynamics, called simple cases (S-I, S-II and S-III). Then there are two regions denoted A-I and A-II, related to the range $0<d_{c c}<1$ for $d_{c}<0$ so that there is coexistence of both the fixed points. Thus the final state depends on the initial condition (i.c. henceforth) of the system, and the structure of 
the related basins of attraction plays a relevant role. These basins may have a simple structure or not, depending on the values of the discontinuity points. The regions A-I and A-II are separated by the straight line, say $(r)$, of equation $d_{c c}=g_{L}\left(d_{c}\right)$, that is

$$
(r): \quad d_{c c}=\alpha d_{c}+(1-\alpha)
$$

which also depends on the parameter $\alpha$.

The region related to more complicated, and interesting, dynamics is associated with the range $0<d_{c}<1$, for which the system has no fixed points in the region denoted $\mathrm{B}$, for $d_{c c}>1$, while the fixed point $x^{* *}=1$ exists in the region denoted $\mathrm{C}$, for $d_{c c}<1$, and it may coexist with a $k$-cycle, $k>1$.

Notice that the qualitative pictures shown in Fig.s 2a, b and $\mathrm{c}$ in Sec.2 correspond to Regions A, B and C, respectively, in the $\left(d_{c}, d_{c c}\right)$-parameter plane presented in Fig.3.

\subsection{Three relatively simple cases}

Let us describe in detail which fixed point is attracting and how the convergence can occur.

Region S-I $\left(d_{c}<0\right.$ and $d_{c c}>1$ : convergence to $\left.x^{*}=0\right)$.

Since it is $g_{U}\left(d_{c c}\right)<d_{c c}$ the fixed point $x^{* *}=1$ does not exist, so that for parameters in this region there exists only a unique steady state $x_{R}^{*}=0$ and the dynamics converge to it, thus $x^{*}$ is globally attracting. Moreover, for any i.c. $x_{0}>0$ the trajectory is monotone decreasing. Differently, for $x_{0}<0$ a change of direction is possible, depending on the value $g_{L}\left(d_{c}\right) \gtreqless 0$. The two different cases are shown in Fig.4a,b. If $g_{L}\left(d_{c}\right)=\alpha d_{c}+(1-\alpha)<0$ then for any i.c.
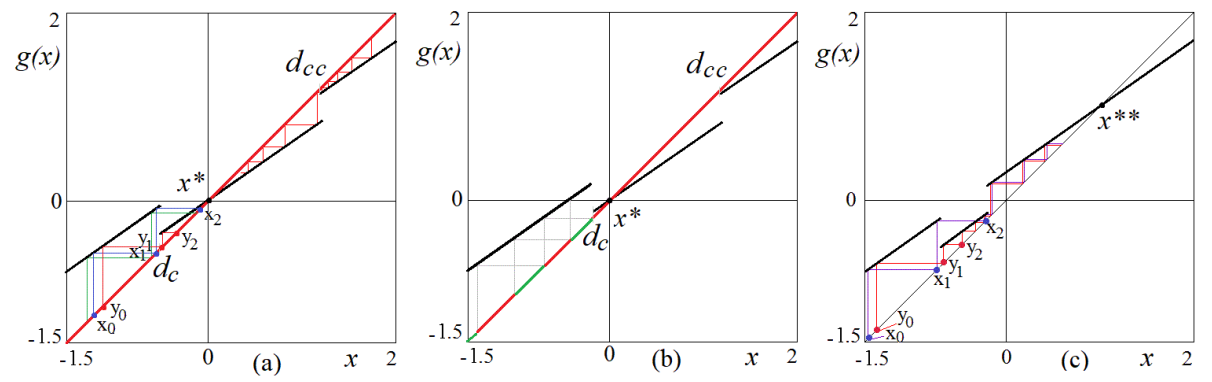

Figure 4: Map $g$ in Region S-I: at $\alpha=0.7, d_{c c}=1.2$ and $d_{c}=-0.5$ in (a), with leapfrogging; $d_{c}=-0.2$ in (b), with overshooting. Map $g$ in Region S-II at $\alpha=0.7, d_{c}=-0.7, d_{c c}=-0.2$ in (c), with leapfrogging.

$x_{0}<0$ the trajectory is monotone increasing. If $g_{L}\left(d_{c}\right)=\alpha d_{c}+(1-\alpha)>0$ then it is possible that a trajectory with negative i.c. overshoots the origin assuming positive values, and after it will decrease to zero. That is, the points belonging to the interval

$$
J_{0}:=\left(0, g_{L}\left(d_{c}\right)\right]=\left(0, \alpha d_{c}+(1-\alpha)\right]
$$


have preimages in the negative side, thus for an i.c. $x_{0} \in g_{L}^{-n}\left(J_{0}\right)$ the trajectory is monotone increasing for the first $n$ periods and monotone decreasing afterwards (see the segments evidenced in Fig.4b). Since $g_{L}^{-1}$ is expanding there are infinitely many preimages (intervals) of $J_{0}$. Moreover, it is possible to have leapfrogging, i.e., considering two initial conditions with $x_{0}<y_{0}<d_{c c}$ then it is possible to have $x_{t}>y_{t}$ after some periods. A necessary condition for this to occur is $g_{L}\left(d_{c}\right)=\alpha d_{c}+(1-\alpha)<0$. An example is shown in Fig.4a.

Region S-II $\left(d_{c}<d_{c c}<0\right.$ : convergence to $\left.x^{* *}=1\right)$.

For parameters in this region there exists only the steady state $x^{* *}=1$. Moreover, from the linearity of the functions we have that any i.c. $x_{0}>1$ has a monotone decreasing trajectory, and since $g(x)>x$ for any $x<1$, the trajectory is monotone increasing for any initial condition $x_{0}<1$. However, as in the previous case it is possible to have leapfrogging. An example is shown in Fig.4c. A necessary condition for this to occur is that at a finite period $k$ the increasing trajectories have $x_{k}<d_{c}<y_{k}$.

Region S-III $\left(1<d_{c}<d_{c c}\right.$ : convergence to $\left.x^{* *}=1\right)$.
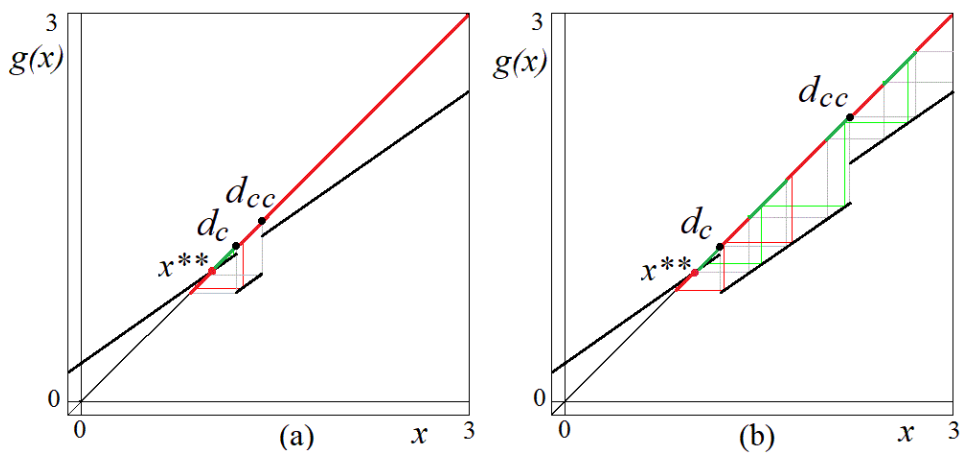

Figure 5: Map $g$ in Region S-III: at $\alpha=0.7, d_{c}=1.2, d_{c c}=1.4(\mathrm{a}) ; \alpha=0.7$, $d_{c}=1.2, d_{c c}=2.2(\mathrm{~b})$.

In this range a trajectory is monotone increasing for any initial condition $x_{0}<1$, while when $x_{0}>1$, being $g(x)<x$ a trajectory starts decreasing but it may ultimately converge to $x^{* *}=1$ increasing. In fact, if $g_{R}\left(d_{c}\right)=\alpha d_{c}<1$, a point from the right side of $x^{* *}=1$ can be mapped to its left side. We can so distinguish between i.c. having a trajectory always on the right side of $x^{* *}=1$ or not. Let $\alpha d_{c}<1$ and

$$
J_{1}:=(0,1) \cap\left(\alpha d_{c}, \alpha d_{c c}\right)
$$

then for $x_{0} \in g^{-n} \circ g_{R}^{-1}\left(J_{1}\right)$ the trajectory is monotone decreasing for the first $n+1$ periods and monotone increasing afterwards; while for i.c. in the complementary sets, that is, for $x_{0} \in(1, \infty) \backslash \cup_{n \geq 0} g^{-n} \circ g_{R}^{-1}\left(J_{1}\right)$ the trajectory is monotone decreasing towards $x^{* *}=1$. In particular, if $\alpha d_{c c}<1$, so that 
$J_{1}=\left(\alpha d_{c}, \alpha d_{c c}\right)$, then the interval $\left(1, d_{c}\right)$ (green segment in Fig.5a) is the only segment whose points have a trajectory monotonically decreasing towards $x^{* *}$ (i.e. $\left.g_{R}^{-1}\left(J_{1}\right)=\varnothing\right)$. Differently, for $\alpha d_{c c}>1$, so that $J_{1}=\left(\alpha d_{c}, 1\right)$, then $g_{R}^{-1}\left(J_{1}\right) \neq \varnothing$ and the interval $\left(1, d_{c}\right)$ has infinitely many intervals as preimages, the points of which have a trajectory monotonically decreasing towards $x^{* *}$ (green segments in Fig.5b).

We can so state the following

Proposition 2 (Globally attracting steady states) For any value of the parameter $\alpha \in(0,1)$, if the parameter point $\left(d_{c}, d_{c c}\right)$ belongs to region $S$-I then the fixed point $x^{*}=0$ is globally attracting; for parameters in the regions $S$-II and S-III the fixed point $x^{* *}=1$ is globally attracting.

\subsection{Two cases in region $\mathrm{A}$}

When the parameters satisfy $d_{c}<0<d_{c c}<1$ then we have two coexisting fixed points, $x^{*}=0$ and $x^{* *}=1$, and the straight line $(r)$ given in (11) separating the two regions A-I and A-II corresponds to a bifurcation in the structure of the related basins of attraction, as described below.

Region A-I: "The low steady state as a poverty trap" for $g_{L}\left(d_{c}\right)=$ $\alpha d_{c}+(1-\alpha)<d_{c c}<1$ (above the line $(r)$ ). The related basins are connected: two intervals separated by the discontinuity point $d_{c c}$, that is:

$$
\mathcal{B}(0)=\left(-\infty, d_{c c}\right) \text { and } \mathcal{B}(1)=\left(d_{c c},+\infty\right)
$$

Considering $x_{0} \in \mathcal{B}(1)$ the trajectory is monotone decreasing for $x_{0}>1$ and monotone increasing for $d_{c c}<x_{0}<1$ (see blue points in Fig.6a). While considering $x_{0} \in \mathcal{B}(0)$ the monotonicity changes depending on $g_{L}\left(d_{c}\right)=\alpha d_{c}+$ $(1-\alpha) \lesseqgtr 0$. If $g_{L}\left(d_{c}\right)<0$ then for $x_{0}<0$ the trajectory is monotone increasing while it is monotone decreasing for $0<x_{0}<d_{c c}$. If $g_{L}\left(d_{c}\right)>0$ then a point on the left side of the steady state $x^{*}=0$ can be mapped to the right side of the fixed point. Thus, for $x_{0} \in \underset{n \geq 0}{\cup} g_{L}^{-n}\left(d_{c}, 0\right)$ the trajectory is monotone increasing; for $0<x_{0}<d_{c c}$ the trajectory is monotone decreasing; for $x_{0} \in$ $g_{L}^{-n}\left(0, \alpha d_{c}+(1-\alpha)\right)$ the trajectory is monotone increasing for the first $n$ periods and monotone decreasing afterwards (see the green points in Fig.6a).

Region A-II: "Reversal of Fortune" for $d_{c}<d_{c c}<g_{L}\left(d_{c}\right)=\alpha d_{c}+(1-$ $\alpha$ ) (below the line $(r)$ ). Now there are always points $x_{0}<d_{c}$ whose trajectories converge to $x^{* *}=1$ so that both the two basins consist of infinitely many intervals. If $x_{0} \in\left(d_{c}, d_{c c}\right)$ then the trajectory converges to $x^{*}=0$. If $x_{0}>d_{c c}$ then the trajectory converges to $x^{* *}=1$. If $x_{0}<d_{c}$ then the trajectory converges either to $x^{*}=0$ or to $x^{* *}=1$. Let

$$
J:=\left(d_{c c}, g_{L}\left(d_{c}\right)\right]=\left(d_{c c}, \alpha d_{c}+(1-\alpha)\right]
$$

then it is

$$
\mathcal{B}(1)=\left(d_{c c},+\infty\right) \cup_{n>0} g_{L}^{-n}(J)
$$



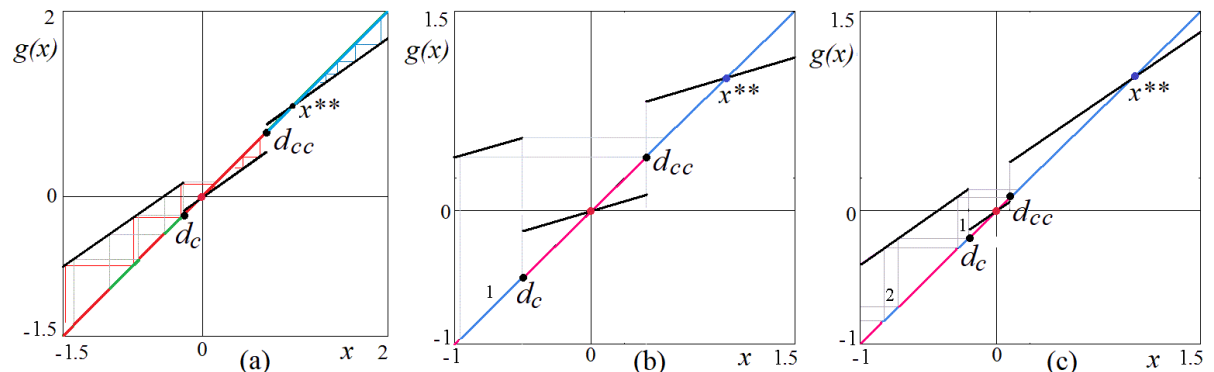

Figure 6: Map $g$ in Region A-I in (a), at $\alpha=0.7, d_{c}=-0.2, d_{c c}=0.7$. Map $g$ in Region A-II in (b,c): at $\alpha=0.3, d_{c}=-0.5, d_{c c}=0.4(\mathrm{~b}) ; \alpha=0.7, d_{c}=-0.2$, $d_{c c}=0.1(\mathrm{c})$.

and

$$
\mathcal{B}(0)=\left(d_{c}, d_{c c}\right) \cup_{n>0} g_{L}^{-n}\left(\left(d_{c}, d_{c c}\right)\right)
$$

The intervals of the two basins $\mathcal{B}(1)$ and $\mathcal{B}(0)$ are alternating, and as already remarked above, since $g_{L}^{-1}$ is expanding, so the infinitely many intervals belonging to the preimages are increasing in width and tend to $-\infty$ (in the state space of the variable $x$ ). Two examples are given in Fig.6b,c.

A point belonging to the interval $g_{L}^{-n}(J)$ takes $n$ iterations to reach the immediate basin of the fixed point $x_{1}^{*}$ from which it converges monotonically to the attracting fixed point.

We can so state the following

Proposition 3 (Coexistence of attracting fixed points) For any value of the parameter $\alpha \in(0,1)$, when the parameter point $\left(d_{c}, d_{c c}\right)$ belongs to regions $A$-I or A-II then the attracting fixed points $x^{*}=0$ and $x^{* *}=1$ coexist. Their basins of attraction are connected and consist in two intervals in region A-I, as given in (12), while they are disconnected and formed by infinitely many alternating intervals in region A-II, as given in (14) and (13).

\subsection{Region B: $n$-cycles, for any $n>1$}

When the parameters satisfy $0<d_{c}<1<d_{c c}$ then neither $x^{*}=0$ nor $x^{* *}=1$ exist as steady states. ${ }^{1}$ Moreover, since it is $d_{c c}>g_{L}\left(d_{c}\right)=\alpha d_{c}+(1-\alpha)$ (i.e. the $\left(d_{c}, d_{c c}\right)$-parameter point is above the straight line $\left.(r)\right)$ we have that the discontinuity point $d_{c c}$ is outside the interval

$$
I=\left[g_{R}\left(d_{c}\right), g_{L}\left(d_{c}\right)\right]=\left[\alpha d_{c}, \alpha d_{c}+(1-\alpha)\right]
$$

\footnotetext{
${ }^{1}$ Notice that also in Asano et al. 2011, the Matsuyama credit cycle model has been investigated for the case of Cobb-Douglas production functions, and for the parameters belonging to Region B.
} 
and from $g_{U}\left(d_{c c}\right)<d_{c c}$ (non existence of the upper fixed point) we can conclude that for any i.c. $x_{0}$ the trajectory enters the absorbing interval ${ }^{2} I$ in (15) in a finite time and continues fluctuating inside $I$. The restriction of the map to this absorbing interval consists in a piecewise linear map with only one discontinuity point, $d_{c}$, and positive slopes $\alpha \in(0,1)$, so any cycle of period $n$ has eigenvalue $\lambda=\alpha^{n}$.

For this kind of maps the dynamics are nowadays well known, and we recall these results for completeness, referring for further details to the existing literature. The first results of the case we are interested in are due to Leonov (1959), (1962) (see also Avrutin et al. 2010, Gardini et al. 2010, where Leonov's technique has been improved). The case with two discontinuity points is considered in Tramontana et al. (2011), but in the present study we are mainly interested in the simplest situation with only one discontinuity point. Other economic applications have been studied leading to maps with a similar structure (examples are in Tramontana et al. 2010, Tramontana et al. 2011, Gardini et al. 2011). In the generic case all the trajectories are converging to a unique attracting $n$-cycle, with $n>1$. Cycles of any period exist and also several kind of cycles of the same period but with different distribution of the periodic points between the right and left side of the discontinuity point. As we shall see, quasiperiodic trajectories may also occur (belonging to an attracting Cantor set in $I$ ), but only for a parameter set of points of zero Lebesgue measure in the parameter plane.

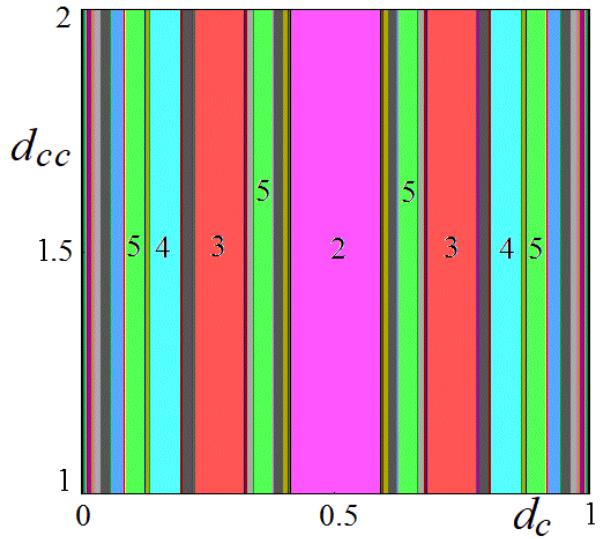

(a)

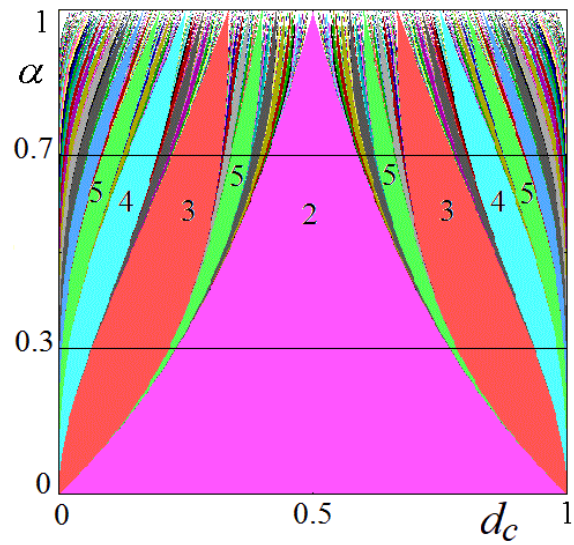

(b)

Figure 7: Case B: (a) the $\left(d_{c}, d_{c c}\right)$-parameter plane for $\alpha=0.7$; (b) the $\left(d_{c}, \alpha\right)$ paramter plane for $d_{c c}>1$.

The periodicity regions associated with different periods are shown in Fig.7 by different colors.

\footnotetext{
${ }^{2}$ Notice that the absorbing interval is a closed interval independently on the definition of the map in the discontinuity point $d_{1}$.
} 
To show that cycles of any period exist let us introduce the symbolic sequence of a cycle in which the symbol $R$ or $L$ corresponds to a periodic point when it belongs to the right or left side, respectively, of the discontinuity point $d_{c}$. A cycle appears and disappears via a BCB related to this discontinuity point. So in the $\left(d_{c}, d_{c c}\right)$-parameter plane we have two BCB curves determining the appearance/disappearance of a cycle. Let us first consider the cycle having the symbolic sequence $L R^{n}, n \geq 1$ (so-called first complexity level cycle) and its periodicity region denoted $\Pi_{L R^{n}}$. Then, when the cycle exists, the periodic point on the left side of $d_{c}$, say $x_{0}$, can be determined as fixed point of the composite function $g_{R}^{n} \circ g_{L}\left(x_{0}\right)=x_{0}$ and we get:

$$
x_{0}=\frac{(1-\alpha) \alpha^{n}}{1-\alpha^{n+1}}
$$

The collision $x_{0}=d_{c}$ leads to the BCB curve of equation

$$
\Phi_{L R^{n}}: d_{c}=\frac{(1-\alpha) \alpha^{n}}{1-\alpha^{n+1}}
$$

In the $\left(d_{c}, d_{c c}\right)$-parameter plane the curves $\Phi_{L R^{n}}$ for various $n \geq 1$ are vertical left side boundaries of the periodicity regions $\Pi_{L R^{n}}$ shown in Fig.7a on the left side of the 2-periodicity region $\Pi_{L R}$. These curves are also shown in Fig.7b in the $\left(d_{c}, \alpha\right)$-parameter plane at a fixed value of $d_{c c}>1$.

The second border collision of this cycle with the discontinuity point $d_{c}$ occurs when the last periodic point $x_{n}$ (the one closest to $d_{c}$ from the right side) merges with $d_{c}$. From $g_{R}^{n-1} \circ g_{L} \circ g_{R}\left(x_{n}\right)=x_{n}$ (or from $g_{R}^{-1}\left(x_{0}\right)=\frac{x_{0}}{\alpha}$ ) we get

$$
x_{n}=\frac{(1-\alpha) \alpha^{n-1}}{1-\alpha^{n+1}}
$$

so that the collision $x_{n}=d_{c}$ leads to the BCB curves of equation

$$
\Phi_{R L R^{n-1}}: d_{c}=\frac{(1-\alpha) \alpha^{n-1}}{1-\alpha^{n+1}}
$$

which are vertical right side boundaries of the periodicity regions $\Pi_{L R^{n}}$ shown in Fig.7a on the left side of the region of the 2-cycle. For increasing $n$ the periodicity regions $\Pi_{L R^{n}}$ become very thin and accumulate to the vertical line $d_{c}=0$.

Similarly we obtain the vertical boundaries of the periodicity regions $\Pi_{R L^{n}}$ of cycles of first complexity level having the symmetric symbolic sequence ${ }^{3} R L^{n}$. From $g_{L}^{n} \circ g_{R}\left(x_{0}\right)=x_{0}$ we get:

$$
x_{0}=\frac{1-\alpha^{n}}{1-\alpha^{n+1}}
$$

so that the collision $x_{0}=d_{c}$ leads to the BCB curves of equation

$$
\Phi_{R L^{n}}: d_{c}=\frac{1-\alpha^{n}}{1-\alpha^{n+1}}=1-\frac{(1-\alpha) \alpha^{n}}{1-\alpha^{n+1}}
$$

\footnotetext{
${ }^{3}$ Let us call two symbolic sequences symmetric if these sequences are obtained from each other exchanging $R$ and $L$ with $L$ and $R$, respectively.
} 
The second border collision of this cycle with the discontinuity point $d_{c}$ occurs when the last periodic point $x_{n}$ merges with $d_{c}$. From $g_{L}^{n-1} \circ g_{R} \circ g_{L}\left(x_{n}\right)=x_{n}$ (or from $\left.g_{L}^{-1}\left(x_{0}\right)=\frac{x_{0}}{\alpha}-\frac{(1-\alpha)}{\alpha}\right)$ we get

$$
x_{n}=\frac{(1-\alpha) \alpha^{n}+1-\alpha^{n-1}}{1-\alpha^{n+1}}
$$

so that the collision $x_{n}=d_{c}$ leads to the BCB curves of equation

$$
\Phi_{L R L^{n-1}}: d_{c}=\frac{(1-\alpha) \alpha^{n}+1-\alpha^{n-1}}{1-\alpha^{n+1}}=1-\frac{(1-\alpha) \alpha^{n-1}}{1-\alpha^{n+1}}
$$

The curves $\Phi_{R L^{n}}$ and $\Phi_{L R L^{n-1}}$ bound the periodicity regions $\Pi_{R L^{n}}$ shown in Fig.7a on the right side of the region of the 2-cycle $\Pi_{L R}$. For increasing $n$ the periodicity regions $\Pi_{R L^{n}}$ become very thin and accumulate to the vertical line $d_{c}=1$.

From the expressions obtained above we can state the following

Proposition 4 (Cycles of the first complexity level) For any value of the parameter $\alpha \in(0,1)$ and $\left(d_{c}, d_{c c}\right)$-parameter point belonging to region $B$, the cycle with symbolic sequence $L^{n}$ exists for

$$
d_{c} \in\left(\frac{(1-\alpha) \alpha^{n}}{1-\alpha^{n+1}}, \frac{(1-\alpha) \alpha^{n-1}}{1-\alpha^{n+1}}\right) \quad\left(\text { region } \Pi_{L R^{n}}\right)
$$

while the cycle with symbolic sequence $R L^{n}$ exists for

$$
d_{c} \in\left(1-\frac{(1-\alpha) \alpha^{n-1}}{1-\alpha^{n+1}}, 1-\frac{(1-\alpha) \alpha^{n}}{1-\alpha^{n+1}}\right) \quad\left(\text { region } \Pi_{R L^{n}}\right)
$$

For any fixed $n>1$, in the $\left(d_{c}, d_{c c}\right)$-parameter plane the two periodicity regions $\Pi_{L R^{n}}$ and $\Pi_{R L^{n}}$ are symmetric with respect to the line $d_{c}=0.5$; the region $\Pi_{L R}$ of the 2-cycle (i.e., for $n=1$ ) is symmetric with respect to $d_{c}=0.5$.

Figure 8 a shows the 2-cycle with symbolic sequence $R L$ and periodic points $x_{0}=g_{R} \circ g_{L}\left(x_{0}\right)$ and $x_{1}=g_{L} \circ g_{R}\left(x_{1}\right)$. It exists and is globally attracting for the parameter values belonging to region $\Pi_{L R}$ defined by $d_{c} \in\left(\frac{\alpha}{1+\alpha}, \frac{1}{1+\alpha}\right)$.

Figure.8b shows the 3 -cycle with symbolic sequence $L R^{2}$ and periodic points $x_{0}<d_{c}<x_{2}<x_{1}$. It exists and is globally attracting for the parameter values belonging to region $\Pi_{L R^{2}}$ defined by $d_{c} \in\left(\frac{(1-\alpha) \alpha^{2}}{1-\alpha^{3}}, \frac{(1-\alpha) \alpha}{1-\alpha^{3}}\right)$. There exists also a 3 -cycle with symbolic sequence $R L^{2}$ and periodic points $x_{1}<x_{2}<d_{c}<x_{0}$, which exists and is globally attracting for the parameter values belonging to region $\Pi_{R L^{2}}$ defined by $d_{c} \in\left(1-\frac{(1-\alpha) \alpha}{1-\alpha^{3}}, 1-\frac{(1-\alpha) \alpha^{2}}{1-\alpha^{3}}\right)$. The two regions $\Pi_{L R^{2}}$ and $\Pi_{R L^{2}}$ are symmetric with respect to $d_{c}=0.5$.

\section{Higher complexity levels}




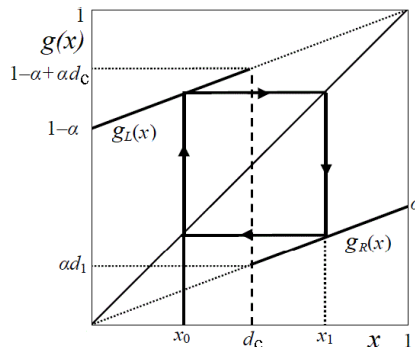

(a)

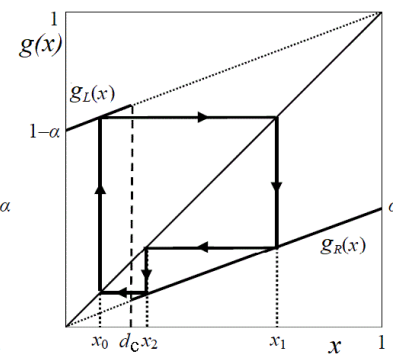

(b)

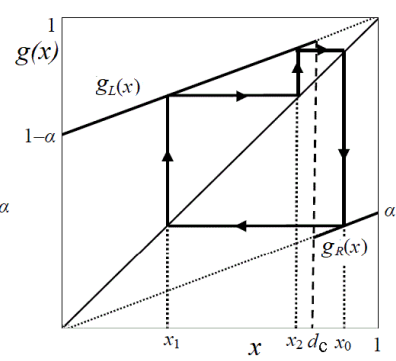

(c)

Figure 8: Map $g$ and its 2-cycle (a); 3-cycle with symbolic sequence $L R^{2}(\mathrm{~b})$; 3 -cycle with symbolic sequence $L^{2} R$ (c).

Up to now we have considered the periodicity regions of the first complexity level cycles having the symbolic sequences $L R^{n}$ and $R L^{n}, n \geq 1$. Besides the periodicity regions of such cycles we can find infinitely many regions associated with cycles of complexity level two, three, and so on, at infinity. These periodicity regions are located according to the following period adding scheme: Between any two consequent regions of the same level $k$, we can detect two infinite families of periodicity regions of complexity level $(k+1)$, accumulating on the two starting regions.

Let us reason for the regions located on the left side of the region $\Pi_{L R}$ of the 2-cycle. Similar reasoning holds by symmetry for the regions located on the right side. For any integer $n \geq 1$, between two consequent periodicity regions of cycles of first level of complexity $\Pi_{L R^{n}}$ and $\Pi_{L R^{n+1}}$ of map $g$, there exist two infinite sequences of periodicity regions of cycles of the second level of complexity, $\Pi_{\left(L R^{n}\right)^{m} L R^{n+1}}$ and $\Pi_{L R^{n}\left(L R^{n+1}\right)^{m}}$, for any $m \geq 1$, which are accumulating on the boundaries of the periodicity regions of first level between which they are located.

To determine the BCB curves bounding the periodicity regions of cycles of second complexity level let us consider the gap between the regions $\Pi_{L R^{n}}$ and $\Pi_{L R^{n+1}}$ for $n \geq 1$, that is $d_{c} \in\left(\frac{(1-\alpha) \alpha^{n}}{1-\alpha^{n+2}}, \frac{(1-\alpha) \alpha^{n}}{1-\alpha^{n+1}}\right)$ for any integer $n \geq 1$. Between the curves $\Phi_{L R^{n}}$ and $\Phi_{R L R^{n}}$ we can consider the composite functions associated with the symbolic sequence of the related colliding points $L R^{n}$ and $R L R^{n}$, or, equivalently, for the gap between $\Pi_{L R^{n}}$ and $\Pi_{L R^{n+1}}$ we define a new map of an interval into itself (the first return map in a neighborhood of the discontinuity point $d_{c}$ ) as follows:

$$
x_{t+1}= \begin{cases}T_{L}\left(x_{t}\right)=g_{R}^{n} \circ g_{L}\left(x_{t}\right) & \text { if } \quad \frac{(1-\alpha) \alpha^{n}}{1-\alpha^{n+2}}<x_{t}<d_{c} \\ T_{R}\left(x_{t}\right)=g_{R}^{n} \circ g_{L} \circ g_{R}\left(x_{t}\right) & \text { if } \quad d_{c}<x_{t}<\frac{(1-\alpha) \alpha^{n}}{1-\alpha^{n+1}}\end{cases}
$$


which can be rewritten as (see Fig.9):

$$
x_{t+1}=\left\{\begin{array}{l}
T_{L}\left(x_{t}\right)=A_{L} x_{t}+B \quad \text { if } \frac{B}{1-A_{R}}<x_{t}<d_{c} \\
T_{R}\left(x_{t}\right)=A_{R} x_{t}+B \quad \text { if } d_{c}<x_{t}<\frac{B}{1-A_{L}}
\end{array}\right.
$$

where $A_{L}=\alpha^{n+1}>A_{R}=\alpha^{n+2}$ and $B=(1-\alpha) \alpha^{n}$.

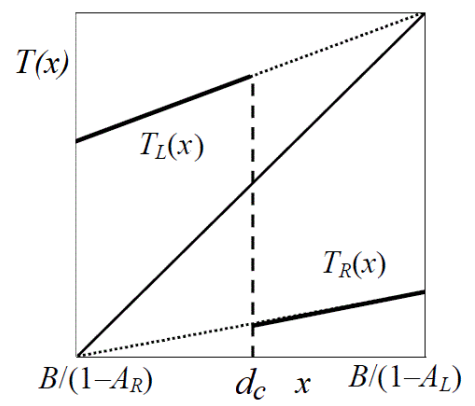

Figure 9: The first return map $T$.

Therefore, following the same procedure as described above for the first level, we can find the $(m+1)$-cycle with the function $T_{L}\left(T_{R}\right)^{m}$, which corresponds to the cycle of map $g$ of period $[(1+n)+m(2+n)]$ with symbolic sequence $L R^{n}\left(R L R^{n}\right)^{m}$, that exists in the periodicity region given by

$$
d_{c} \in\left(\frac{B\left(1-A_{R}^{m+1}\right)}{\left(1-A_{R}\right)\left(1-A_{L} A_{R}^{m}\right)}, \frac{B\left[1-A_{R}^{m}+A_{L}\left(1-A_{R}\right) A_{R}^{m-1}\right]}{\left(1-A_{R}\right)\left(1-A_{L} A_{R}^{m}\right)}\right)
$$

leading to the equations of the related BCB curves:

$$
\begin{gathered}
\Phi_{T_{L} T_{R}^{m}}: d_{c}=B \frac{\left(1-A_{R}^{m+1}\right)}{\left(1-A_{R}\right)\left(1-A_{L} A_{R}^{m}\right)} \\
\Phi_{T_{R} T_{L} T_{R}^{m-1}}: d_{c}=B \frac{1-A_{R}^{m}+A_{L}\left(1-A_{R}\right) A_{R}^{m-1}}{\left(1-A_{R}\right)\left(1-A_{L} A_{R}^{m}\right)}
\end{gathered}
$$

As $m \rightarrow \infty$ these regions accumulate to the right boundary of $\Pi_{L R^{n+1}}$.

Similarly, we can find the $(m+1)$-cycle with the function $\left(T_{L}\right)^{m} T_{R}$, which corresponds to the cycle of map $g$ of period $[(2+n)+m(1+n)]$ with symbolic sequence $\left(L R^{n}\right)^{m} L R^{n+1}$, that exists in the periodicity region given by

$$
d_{c} \in\left(\frac{B\left(1-A_{L}^{m+1}\right)}{\left(1-A_{L}\right)\left(1-A_{R} A_{L}^{m}\right)}, \frac{B\left[1-A_{L}^{m}+A_{R}\left(1-A_{L}\right) A_{L}^{m-1}\right]}{\left(1-A_{L}\right)\left(1-A_{R} A_{L}^{m}\right)}\right)
$$

leading to the equations of the related BCB boundaries. As $m \rightarrow \infty$ these regions accumulate to the right boundary of $\Pi_{L R^{n}}$.

Thus, there exist two infinite sequences of periodicity regions of cycles of the second complexity level, related to cycles with symbolic sequence $L R^{n}\left(L R^{n+1}\right)^{m}$ 
and $\left(L R^{n}\right)^{m} L R^{n+1}$ for any integer $m \geq 1$ whose periodicity regions are accumulating as $m \rightarrow \infty$ to $\Pi_{L R^{n+1}}$ and $\Pi_{L R^{n}}$, respectively. This procedure can be repeated infinitely many times: between any two consequent regions of cycles of the $k$-th level of complexity, we can detect two infinite families of periodicity regions of complexity level $(k+1)$, accumulating towards the two starting regions.

The union of all the disjoint periodicity regions constructed in this way does not cover the entire interval $d_{c} \in(0,1)$. The remaining set of values of $d_{c}$ is a set of measure zero. For the parameter values belonging to this set the trajectory is quasiperiodic, dense in the invariant set, which is a Cantor set (see Hao 1989, Keener 1980, Gardini et al. 2010).

Figure 10 shows two bifurcation diagrams, tracing the orbit of stable cycles as a function of $d_{c} \in(0,1)$ along the two horizontal lines in Fig.7b, at the values $\alpha=0.3$ and $\alpha=0.7$.

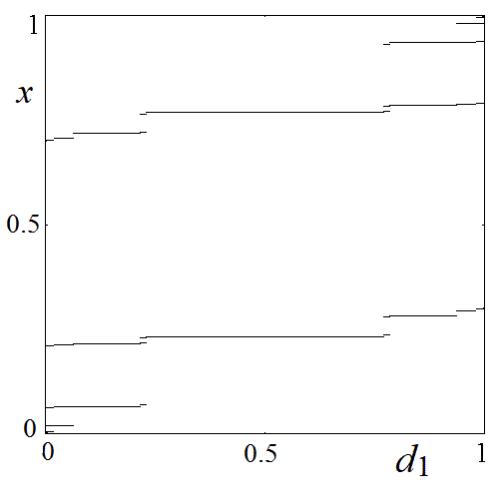

(a)

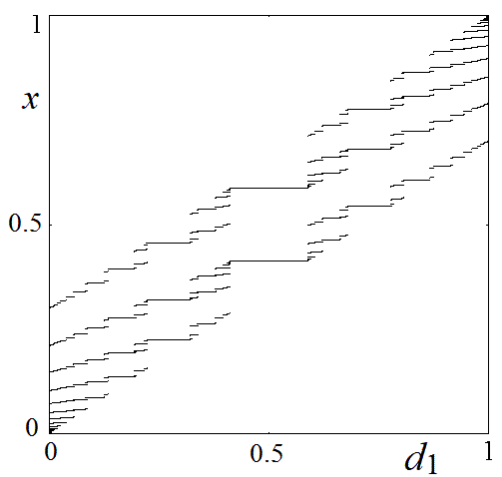

(b)

Figure 10: 1D bifurcation diagrams for (a) $\alpha=0.3, d_{c c}=1.5$; (b) $\alpha=0.7$, $d_{c c}=1.5$.

\section{The rotation (or winding) number}

Along each orbit of a stable cycle we can calculate what fraction of the periods the economy is in an expansionary stage (that is, on the left side of $\left.d_{c}\right)$. For the $k$-cycles, along which the periodic orbit visits $p$ times the $L$ side and $k-p$ times the $R$ side, we can associate its rotation number, $\omega:=\frac{p}{k}$. For example, considering cycles of first level of complexity we have

$$
\omega=\frac{1}{1+n} \text { for } L R^{n} \text { and } \omega=\frac{n}{1+n} \text { for } R L^{n}
$$

For cycles of second level of complexity between $L R^{n+1}$ and $L R^{n}$ :

$$
\omega=\frac{1+m}{(1+n)+m(2+n)} \text { for } L R^{n}\left(L R^{n+1}\right)^{m}
$$


and

$$
\omega=\frac{1+n m}{(2+n)+m(1+n)} \text { for }\left(R L^{n}\right)^{m} L R^{n+1}
$$

and so on. More generally, between two periodicity regions of cycles with rotation numbers $\frac{p_{1}}{k_{1}}$ and $\frac{p_{2}}{k_{2}}$ we can find the periodicity region of cycles with the rotation number given by

$$
\frac{p_{1}}{k_{1}} \oplus \frac{p_{2}}{k_{2}}=\frac{p_{1}+p_{2}}{k_{1}+k_{2}}
$$

where $\oplus$ denotes an operation known as Farey summation rule. Furthermore, the rotation number can be expressed as a function of $d_{c}, \omega\left(d_{c}\right)$. It is a continuous non-decreasing function which goes up from zero to one, and with zero derivative almost everywhere. That is, it is not absolutely continuous. It is a singular (Cantor) function, often referred to as the Devil's staircase. Fig.11 shows the two graphs of $\omega\left(d_{c}\right)$ along the two horizontal lines marked in Fig.7b, at the values $\alpha=0.3$ and $\alpha=0.7$, related to the one-dimensional bifurcation diagrams in Fig.10.

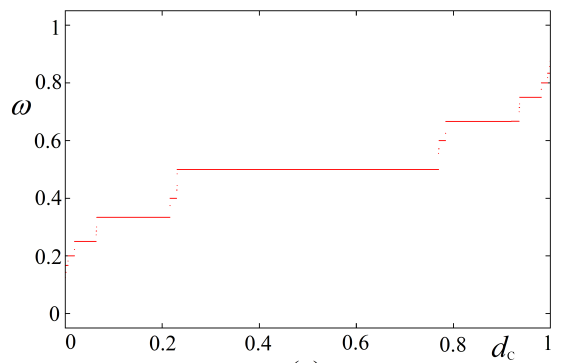

(a)

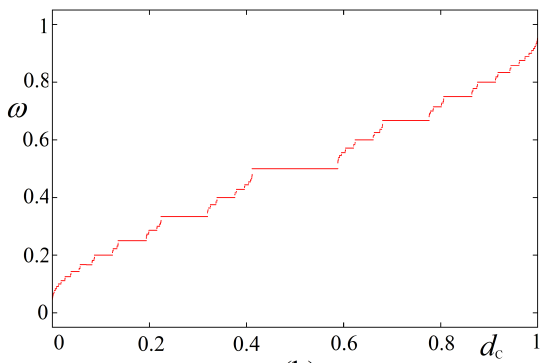

(b)

Figure 11: The rotation number $\omega\left(d_{c}\right)$ for $\alpha=0.3, d_{c c}=1.5(\mathrm{a}) ; \alpha=0.7$, $d_{c c}=1.5(\mathrm{~b})$.

\subsection{Region C: $x^{* *}$ and coexistence with $k$-cycles, for any $k>1$}

When the parameters belong to region $\mathrm{C}\left(0<d_{c}<d_{c c}<1\right)$, then $x^{* *}=1$ exists, and it is the unique steady state. Furthermore, all the stable cycles discussed above, for parameters in region $\mathrm{B}$, survive as long as $d_{c c}$ is greater than the rightmost periodic point along each orbit. So for some i.c. the trajectory eventually crosses over $d_{c c}$ and converges to $x^{* *}=1$, while for other i.c. the trajectory converges to a $k$-cycle. As soon as $d_{c c}$ collides with the largest periodic point of a cycle, the cycle is destroyed. However, this bifurcation occurs only when the parameters are below the straight line $(r)$. This explains the lower boundary of the periodicity regions, as shown in Fig.12. 


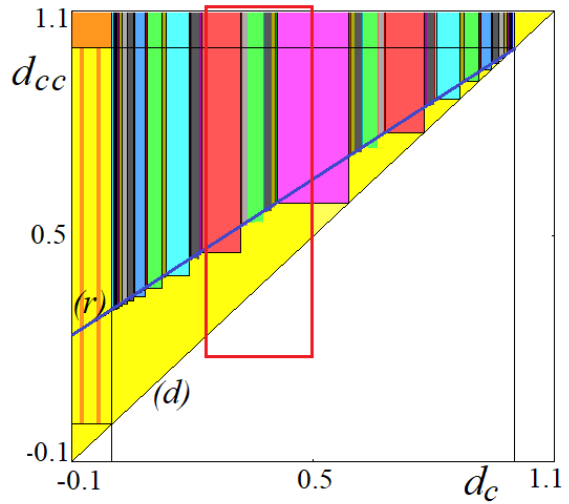

(a)

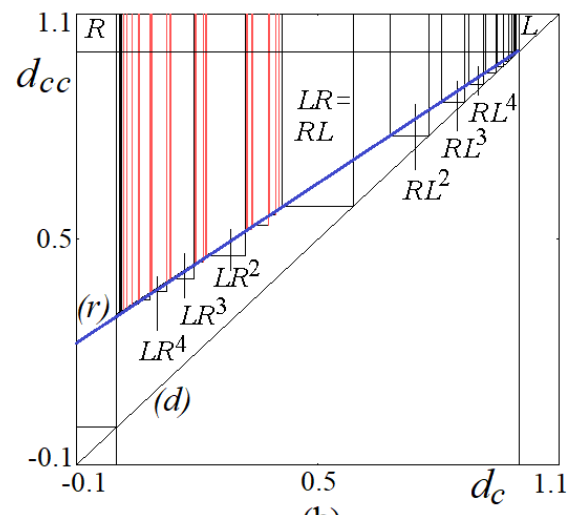

(b)

Figure 12: (a) 2D bifurcation diagram in the $\left(d_{c}, d_{c c}\right)$-parameter plane at $\alpha=$ 0.7; The marked rectangle is shown enlarged in Fig.13a. In (b) the BCB curves are drawn in black and red for the first and second complexity level, respectively.

In this figure, between the periodicity regions $\Pi_{L R}$ and $\Pi_{L R^{2}}$, a few regions of the cycles of the second level of complexity can be seen. The portion in the rectangle is enlarged in Fig.13.

\section{Lower boundaries}

As commented above, all the vertical strips of the periodicity regions existing in region $\mathrm{B}$ for $d_{c c} \geq 1$ also extend to region $\mathrm{C}$, for $d_{c c}<1$ (coexisting with the fixed point $x^{* *}=1$ ) and now the appearance/disappearance of a $k$ cycle, with $k>1$, can occur via collision of any one of the two discontinuity points. Since any i.c. $x_{0}>d_{c c}$ converges to the fixed point $x^{* *}=1$ we can also state that all the periodic points of a $k$-cycle, $k>1$, must be located on the left side of the discontinuity point $d_{c c}$. Thus, the BCB related to the appearance/disappearance of a $k$-cycle, $k>1$, can occur via collision with $d_{c}$ both from the left and right side, leading to the curves already determined for region $\mathrm{B}$, that is, vertical straight lines in Fig.13, plus via collision with $d_{c c}$ from the left side, that is with the maximal periodic point of the cycle. The later collision corresponds to the BCB leading to the third boundary of each periodicity region. So the horizontal line bounding each periodicity regions from below in Fig.13 is related to the collision of the maximal periodic point with the second discontinuity point $d_{c c}$. For the family of cycles having the symbolic sequence $L R^{n}$, the periodic point which may collide with $d_{c c}$ has the symbolic sequence $R^{n} L$, thus from $g_{L} \circ g_{R}^{n}\left(x_{1}\right)=x_{1}$, or from $x_{1}=g_{L}\left(x_{0}\right)$ where $x_{0}$ is given in (16), we get:

$$
x_{1}=\frac{1-\alpha}{1-\alpha^{n+1}}
$$




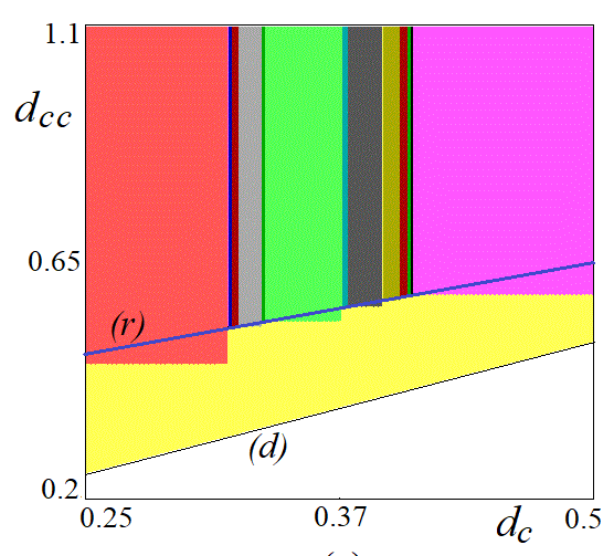

(a)

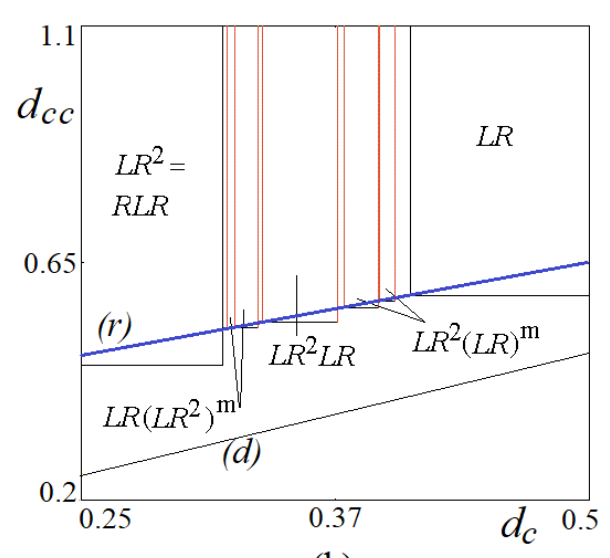

(b)

Figure 13: (a) An enlargement of the rectangle marked in Fig.12a; (b) The related bifurcation boundaries and symbolic sequences.

so that the collision $x_{1}=d_{c c}$ leads to the BCB curves of equation

$$
d_{c c}=\frac{1-\alpha}{1-\alpha^{n+1}}
$$

which are horizontal boundaries of the periodicity regions $\Pi_{L R^{n}}$ shown in Fig.12 (for $n=1, \ldots, 11$ ), on the left side of the region $\Pi_{L R}$ of the 2-cycle.

For the family of cycles having the symbolic sequence $R L^{n}$, the periodic point which may collide with $d_{c c}$ (i.e. the maximum of the periodic points) is the point $x_{0}$ already determined in (20), so that the collision $x_{0}=d_{c c}$ leads to the $\mathrm{BCB}$ curves of equation

$$
d_{c c}=\frac{1-\alpha^{n}}{1-\alpha^{n+1}}
$$

which are horizontal lines of the periodicity regions $\Pi_{L R^{n}}$ shown in Fig.12 (for $n=1, \ldots, 11$ ), on the right side of the region $\Pi_{L R}$ of the 2 -cycle. It is easy to see that the following properties hold, which state that for each periodicity region, the value of $d_{c c}$ characterizing the lower boundary of the existence region $\Pi_{L R^{n}}$ is obtained by the intersection of the left boundary of the region with the straight line $(r)$ :

Proposition 5 The $B C B$ curve given in (25) (resp. in (26)) intersects the $B C B$ curve $\Phi_{L R^{n}}$ given in (17) (resp. the BCB curve $\Phi_{L R L^{n-1}}$ given in (23)) in a point of the straight line $(r)$ given in (11). That is:

$$
\left(\frac{(1-\alpha) \alpha^{n}}{1-\alpha^{n+1}}, \frac{1-\alpha}{1-\alpha^{n+1}}\right) \in(r)
$$


and

$$
\left(\frac{1-\alpha^{n+1}-(1-\alpha) \alpha^{n-1}}{1-\alpha^{n+1}}, \frac{1-\alpha^{n}}{1-\alpha^{n+1}}\right) \in(r)
$$

The properties listed above hold also for all the other periodicity regions of higher complexity level. Thus the lower boundary (determined by the collision of the periodic point closest to the discontinuity point $d_{c c}$ ) can be also determined simply by using the straight line $(r)$. Notice that since the codimension-2 BCB point determined in Proposition 5 is the left boundary of the related periodicity region (vertical boundary of the strip in the parameter plane $\left(d_{c}, d_{c c}\right)$ ), we have that in the region below the straight line $(r)$ we can find parameters for which there is coexistence between the stable fixed point $x^{* *}=1$ with a cycle of any period and any symbolic sequence of the Farey tree starting from the symbols $L$ and $R$. Moreover, another property is peculiar of the periodicity regions of first complexity level on the right side of the 2-cycle region $\Pi_{L R}$, as stated in the following

Proposition 6 In region $C$, below the straight line $(r)$ we can find parameters for which the stable fixed point $x^{* *}=1$ coexists with a cycle of any period and any symbolic sequence of the Farey tree starting from the symbols $L$ and $R$. In particular, the horizontal $B C B$ curves given in (26) intersect the rightmost boundary of the same periodicity regions, that is the $B C B$ curves $\Phi_{R L^{n}}$ given in (21), in a point belonging to the diagonal $d$ of equation $d_{c}=d_{c c}$.

An example of the periodicity regions in a different parameter space is shown in Fig.14, at fixed $d_{c c}=0.8$, showing the regions in the $\left(d_{c}, \alpha\right)$-parameter plane.

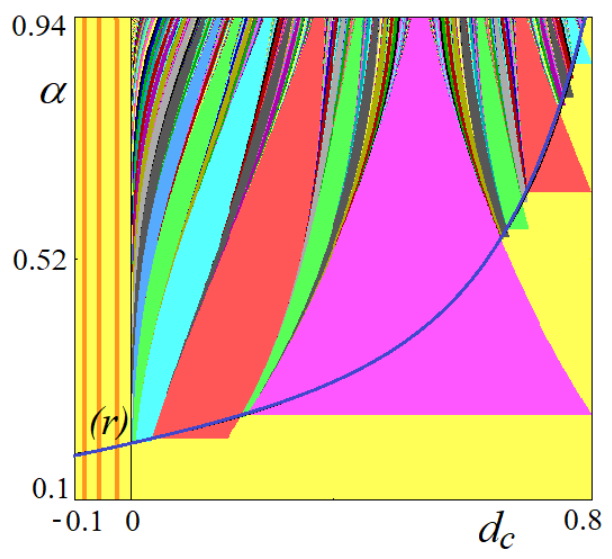

Figure 14: 2D bifurcation diagram in the $\left(d_{c}, \alpha\right)$-parameter plane for $d_{c c}=0.8$. All the points in this figure for $0<d_{c}=0.8$ belong to Region C.

From the properties described up to now, we have that for a point $\left(d_{c}, d_{c c}\right)$ in region $\mathrm{C}$ above the straight line $(r)$ we always have coexistence between $x^{* *}=1$ 
with some $k$-cycle. While for a point $\left(d_{c}, d_{c c}\right)$ in region $\mathrm{C}$ below the straight line $(r)$ we may have coexistence between $x^{* *}=1$ with some $k$-cycle, or $x^{* *}=$ 1 is globally attracting (and this occurs in the yellow region of Fig.14 below the colored periodicity regions of $k$-cycles). Thus, in the cases of coexistence we are interested in determining the related basins of attraction. We have different structure of the basins depending on the two cases. That is, when there is bistability, then the structure of the basins is different depending on the condition determined with the straight line $(r)$ given in (11), and the comments are similar to those already done for region A.

Case C-I: "Cycles as a poverty trap" for $g_{L}\left(d_{c}\right)=\alpha d_{c}+(1-\alpha)<$ $d_{c c}<1$ (above the straight line $(r)$ ). In such a case the absorbing interval $I=\left[g_{R}\left(d_{c}\right), g_{L}\left(d_{c}\right)\right]=\left[\alpha d_{c}, \alpha d_{c}+(1-\alpha)\right]$ in $(15)$ is on the left side of the discontinuity point $d_{c c}$ and any i.c. $x_{0}<d_{c c}$ converges to a $k$-cycle for some $k>1$. Thus we always have coexistence and the related basins are connected, consisting in two disjoint intervals, that is $\mathcal{B}(1)=\left(d_{c c},+\infty\right)$ and $\mathcal{B}(\mathcal{C})=\left(-\infty, d_{c c}\right)$, where $\mathcal{B}(\mathcal{C})$ denotes the basin of a cycle. An example is shown in Fig.15a.

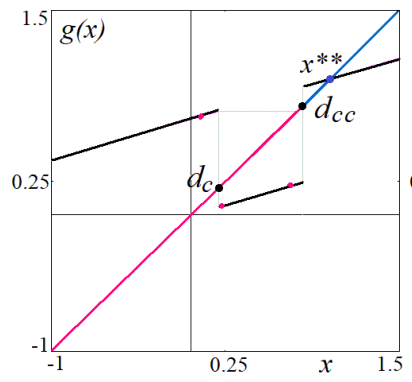

(a)

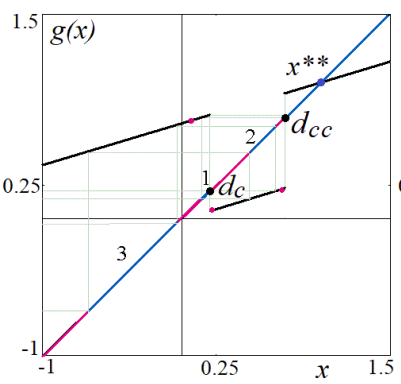

(b)

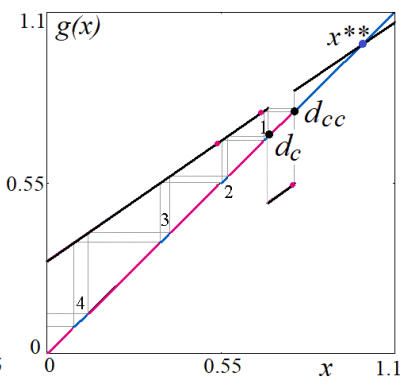

(c)

Figure 15: Map $g$ in Region $C$. (a) Case C-I at $\alpha=0.3, d_{c}=0.2, d_{c c}=0.8$, with disconnected basins; (b) Case C-II at $\alpha=0.3, d_{c}=0.2, d_{c c}=0.74$; (c) Case C-II at $\alpha=0.7, d_{c}=0.7, d_{c c}=0.78$.

Case C-II: "Growth miracle" for $d_{c}<d_{c c}<g_{L}\left(d_{c}\right)=\alpha d_{c}+(1-\alpha)$ (below the straight line $(r)$ ). In that case we may have coexistence and the related basins are not connected, consisting in infinitely many intervals, or $x^{* *}=$ 1 is globally attracting.

Consider $\mathcal{B}^{0}(1)=\left(d_{c c},+\infty\right)$ which is the immediate basin of attraction for $x^{* *}$. Then, for any $n>1, \mathcal{B}^{n}(1)=g^{-n}\left(\left(d_{c c}, g_{L}\left(d_{c}\right)\right)\right.$ is the set of initial conditions from which, after $n$ iterations, the trajectory enters the immediate basin of attraction of $x^{* *}$, so that the basin of attraction of $x^{* *}=1$ is given by $\mathcal{B}(1)=\underset{n \geq 0}{\cup} \mathcal{B}^{n}(1)$. Clearly, if $x^{* *}=1$ is globally attracting then $\mathcal{B}(1)=\mathbb{R}$, otherwise the basin of attraction for the cycle is the complementary set, $\mathcal{B}(C)=$ $\mathbb{R} \backslash C l(\mathcal{B}(1))$, where $C_{l}(\mathcal{B}(1))$ denotes the closure of the basin of attraction of the fixed point $x^{* *}=1$.

When $x^{* *}=1$ is globally attracting then for all $x_{0}<d_{c c}$ the trajectory 
eventually crosses over $d_{c c}$ after which the convergence is monotone increasing. However, the equilibrium trajectory may change its direction many times (unlike in the other case S-III, where it changes its direction at most once).

When $x^{* *}=1$ coexists with a $k$-cycle, then the related basins are disconnected:

$$
\mathcal{B}(1)=\left(d_{c c},+\infty\right) \cup_{n>0} g^{-n}\left(d_{c c}, g_{L}\left(d_{c}\right)\right), \quad \mathcal{B}(\mathcal{C})=R \backslash C_{l}(\mathcal{B}(1))
$$

and the structure of $\mathcal{B}^{n}(1)=g^{-n}\left(\left(d_{c c}, \alpha d_{c}+(1-\alpha)\right)\right.$ can be quite complicated. The intervals of the two basins are always alternating, and due to the fact that the local inverses $g_{L}^{-1}$ and $g_{R}^{-1}$ are expanding, the infinitely many intervals are increasing in width and tend to $-\infty$ (in the state space of the variable $x)$. It is clear that a point belonging to the interval $g^{-n}\left(d_{c c}, g_{L}\left(d_{c}\right)\right)$ takes $n$ iterations to reach the immediate basin of the fixed point $x^{* *}=1$ from which it will converge monotonically to the attracting fixed point. We notice that for parameters in region A-II it is always $g^{-n}\left(d_{c c}, g_{L}\left(d_{c}\right)\right)=g_{L}^{-n}\left(d_{c c}, g_{L}\left(d_{c}\right)\right)$ which is necessarily a unique segment while now, for parameters in region $\mathrm{C}$, the preimages may be no longer unique. The existence of two distinct preimages occurs whenever an interval intersects the interval $\left(\alpha d_{c}, \alpha d_{c c}\right)$, as each point $z$ of this interval has two distinct rank-1 preimages, both with $g_{L}^{-1}$ and $g_{R}^{-1}$, and we have $g_{R}^{-1}(z) \in\left(d_{c}, d_{c c}\right)$.

We remark that it may happen that an intersection with the interval $\left(\alpha d_{c}, \alpha d_{c c}\right)$ occurs, as in the example shown in Fig.15b, or not, as in the example shown in Fig.15c. This figure shows the coexistence of a 3-cycle with the fixed point $x^{* *}=1$ and the intervals from which the orbit will eventually escape converging to $x^{* *}=1$.

\section{Conclusions}

A regime-switching model of credit frictions, proposed by Matsuyama (2007), can display a wide array of dynamical behavior. In this paper, we showed a complete characterization of the dynamic behavior of this model for the CobbDouglas case, which makes the dynamical system piecewise linear. Among others, we showed,

- How overshooting, leapfrogging and reversal of fortune can occur.

- How stable cycles of any period can emerge.

- Along each stable cycle, how the economy alternates between the expansionary and contractionary phases.

- How asymmetry of cycles (the fraction of time the economy is in the expansionary phase) varies with the credit frictions parameters.

- How the economy may fluctuate for a long time at a lower level before successfully escaping from the poverty, etc.

The analysis was done for a restrictive set of assumptions, with only two projects and two switching points, because it is sufficient to create a rich array of dynamics with a relatively few parameters. Obviously, with more projects and more switching points, the model would generate even richer behaviors. 
What simplifies the analysis is the discontinuity and piecewise linearity of the dynamics. Similar results can be numerically obtained with a piecewise smooth discontinuous map and also when the discontinuous piecewise linear or piecewise smooth map is approximated by a continuous map with very steep slopes.

We also hope that the analytical tool used in this paper should be useful for many other dynamic economic models.

\section{Acknowledgements}

I. Sushko thanks the University of Urbino for the hospitality experienced during her stay there as a Visiting Professor. K. Matsuyama is also grateful to the University of Urbino for the hospitality during his visit.

\section{References}

[1] Asano, T., Kunieda, T., Shibata, A.: Complex behaviour in a piecewise linear dynamic macroeconomic model with endogenous discontinuity. Journal of Difference Equations and Applications (2011). http://doi:10.1080/10236198.2011.601301

[2] Avrutin, V., Schanz, M., Gardini, L.: Calculation of bifurcation curves by map replacement. Int. J. Bifurcation and Chaos 20(10), 3105-3135 (2010)

[3] Diamond, P.: Government debt in a neoclassical growth model. American Economic Review 55, 1126-1150 (1965)

[4] Gardini, L., Tramontana, F., Avrutin, V., Schanz, M.: Border Collision Bifurcations in 1D PWL map and Leonov's approach. Int. J. Bifurcation and Chaos 20(10), 3085-3104.(2010)

[5] Gardini, L., Merlone, U., Tramontana, F.: Inertia in binary choices: continuity breaking and big-bang bifurcation points. Journal of Economic Behavior and Organization 80, 153-167 (2011)

[6] Hao, B.-L.: Elementary Symbolic Dynamics and Chaos in Dissipative Systems. World Scientific, Singapore (1989)

[7] Keener, J.P.: Chaotic behavior in piecewise continuous difference equations. Trans. Amer. Math. Soc. 261(2), 589-604 (1980)

[8] Leonov, N.N.: On a pointwise mapping of a line into itself. Radiofisica 2(6), 942-956 (1959)

[9] Leonov, N.N.: On a discontinuous pointwise mapping of a line into itself. Dolk. Acad. Nauk SSSR 143(5), 1038-1041 (1962)

[10] Matsuyama, K.: Credit Traps and Credit Cycles. The American Economic Review, 503-316.(2007) 
[11] Tramontana, F., Gardini, L., Ferri, P.: The dynamics of the NAIRU model with two switching regimes. Journal of Economic Dynamic and Control 34, 681-695 (2010)

[12] Tramontana, F., Gardini, L., Agliari, A.: Endogenous cycles in discontinuous growth models. Mathematics and Computers in Simulation 81, 16251639 (2011)

[13] Tramontana, F., Gardini, L., Avrutin, V., Schanz, M.: Period Adding in Piecewise Linear Maps with Two Discontinuities. Int. J. Bifurcation and Chaos 22, 3 (2012). http://doi:10.1142/S021812741250068X 